\title{
Paper
}

\section{Development of the SR Based Auto-tuning Image Enhancement System and Evaluation of the Enhanced Image Quality using PQM}

\author{
NORIKO KOJIMA ${ }^{* \dagger} \quad$ Member, \\ NaOfumi Matsumoto ${ }^{\dagger}$ Member, \\ BIKASH LAMSAL \\ Member \\ Mitsuo YAMASHIRO ${ }^{\dagger}$ Non-member
}

(Received November 29, 2018, revised March 14, 2019)

\begin{abstract}
This paper proposes the development and evaluation of an auto-tuning stochastic resonance (SR) for image enhancement on images under various illumination conditions. Perceptual Quality Metric (PQM) is used for evaluating and quantifying the image quality. The current process is developed being based on our previous works related to the image enhancement by using the manual tuning stochastic resonance. The process was performed by adding the random noise and threshold in an image. The process works properly in the dark and very low contrast images as well as bright images. This image enhancement system works on dark and bright images as well. The system was tested with the face detection algorithm on the dark and illumination variant images. In this paper, we present the idea of auto-tuning of the SR iteration with random noise and threshold value 0 by using the process related to the histogram calculation, mean and median. In this paper, we performed various experiments on object and human detection as well under different conditions and confirmed the effectiveness of our auto-tuning SR based image enhancement algorithm. Finally, we conducted experiments using Perceptual Quality Metric which is an image quality metric to apply the proposed algorithm in various fields.
\end{abstract}

Keywords: Image enhancement, Stochastic resonance, Parameter auto-tuning, Perceptual quality metric

\section{Introduction}

In an image obtained from a consumer based digital camera, it is very difficult to discriminate an object to be detected from an image due to the several shooting situations under various illumination conditions such as lightening, back lightening, darkness, brightness, halation, flare, ghost, etc. To detect the presence of the proper object from such type of image is a difficult task. To overcome such type of problems, contrast adjustment process is being used from several years. This is a process of expanding the width of the histogram and converting the density for an image which is difficult for vision due to the absence of the difference in the brightness of the image.

But, there exists a lot of case images where the image cannot be enhanced by the contrast adjustment process, which has been clarified in our previous researches related to the face and human body detection on dark and illumination variant images [1] [3]. The another process for image enhancement on such type of images is image sharpening and filtering, that enhances high frequency components of edges and details for optically degraded images. But, this process focus on the contrast adjustment method described before.

\footnotetext{
*n.kojima0904@gmail.com

$\dagger$ Ashikaga University, 268-1, Omae-cho, Ashikaga, Tochigi, Japan, 3268558

$\dagger$ KAJIMA Corporation, Technical Research Institute2-19-1, Tobitakyu, Chofu, Tokyo, Japan, 182-0036
}

The use of stochastic resonance (SR) as an idea of enhancing an image with strict lighting conditions and clear the detection target is shown in the various researches [4] [6]. But, all of these researches do not focus on dark and illumination variant images. Also, doesn' $t$ deal with the auto-tuning parameters in SR. Therefore, there remains a margin for considering the image enhancement algorithm based on SR.

In our previous researches, we proposed an image enhancement algorithm based on SR [1] [3]. The SR parameters were tuned manually in our previous works. Upgrading our previous researches, we are proposing an image enhancement method that auto tunes the SR parameters in order to solve the difficult problem of feature detection and recognition due to the influence of contrast such as illumination variant, dark and bright image.

Our current method of auto-tuning SR is tested by using the dark and illumination variant images used in various fields. Once the image is enhanced, it is then combined with face detector or human detector for detecting the presence of face or human in the given image. Our previous works were focused on the face detection, so the images were experimented by combining with the face detector. We are using the Open CV based human detector for the experimentation. The enhanced images are also compared with the contrast adjustment process for clarifying the effectiveness of our proposed method.

Experiments using face and human detectors showed the 

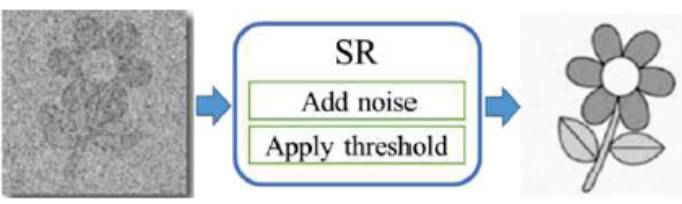

Figure 1: Applying SR idea for image enhancement.

effectiveness of SR for image enhancement. However, a method of evaluating images without using these detectors is also necessary. We conducted experiments using Perceptual Quality Metric (PQM) which is an image quality metric as an evaluation method to apply the proposed method in various fields.

\section{Proposing an image enhancement algorithm using SR}

The proposed method is structured in various steps. The stochastic resonance (SR) process is being implemented and modified for the image enhancement on dark and illumination variant images under strict shooting situations. The basic SR algorithm along with our modified process for image enhancement are briefly described below.

2.1 Basic principle of SR The basic principle of SR was developed by Benzi [7] The principle of SR is to amplify the amplitude of the signal by adding appropriate Gaussian white noise to the original signal and increase the probability that it will exceed the threshold at peak time. SR was first developed to work on the field of the signal processing, but we have applied this process in the field of image processing for image enhancement as shown in the Fig. 1. That is, it is intended to be able to extract information hidden by noise in the image. The noise and threshold is applied in a proper amount for getting the proper result. The noise value and the threshold value is manually tuned in this process.

In this research, SR is used for image scan data and Gaussian white noise is generated using Ziggurat algorithm. So far, we proposed a basic idea using SR to detect faces from

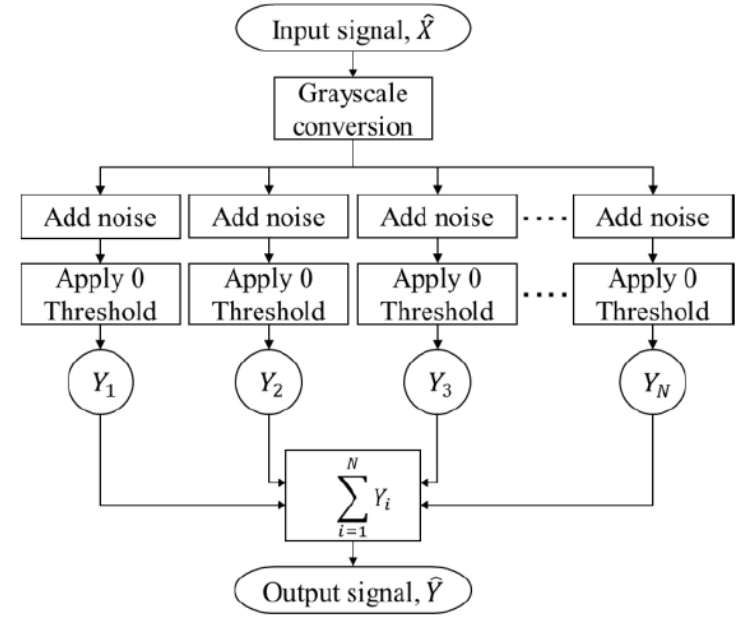

Figure 2: Flow chart of the Collins type SR for image enhancement.

images with severe photographing condition, and clarified the effectiveness by some experiments [1] [2].

2.2 Collins type SR for image enhancement This technique was implemented by Collins et al. [8]. for the time series signal processing. We adopted this idea for the image enhancement by following the Pulse density modulation [9]. This method is a modification of the original SR to parallel summation processing, where the tuning of the $N$ ( $N_{t}$ une $)$ is required. The $N$ is tuned manually in this process by checking the enhanced level of the image. Fig. 2 shows the algorithm. In the Fig. 3, the preliminary experiment was carried out shows the flow for this.by using the noise level at 3 and threshold value 0 [2]. In the original image $\left(N_{\text {tune }}=0\right)$, nothing is seen clearly, as the $N_{\text {tune }}$ level increases, the people in the image along with the persons in the background are confirmed. From the histogram, we can see that the histogram spreads in a bright direction as $N_{\text {tune }}$ increases. Depending on the situation of the original image, when $N_{\text {tune }}$ reaches a certain value, face detection of the person in front is possible. In this image, the face is detected at
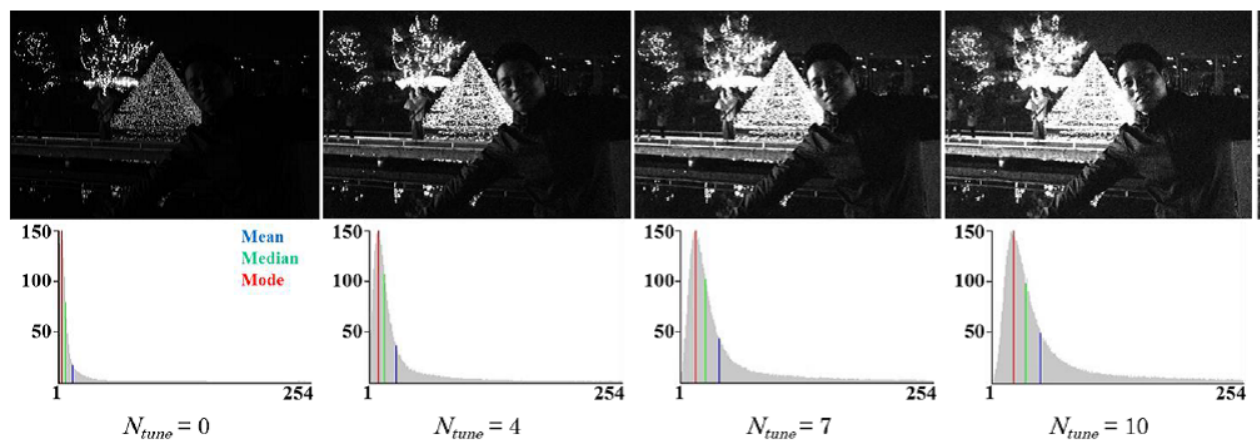

$N_{\text {tune }}=7$
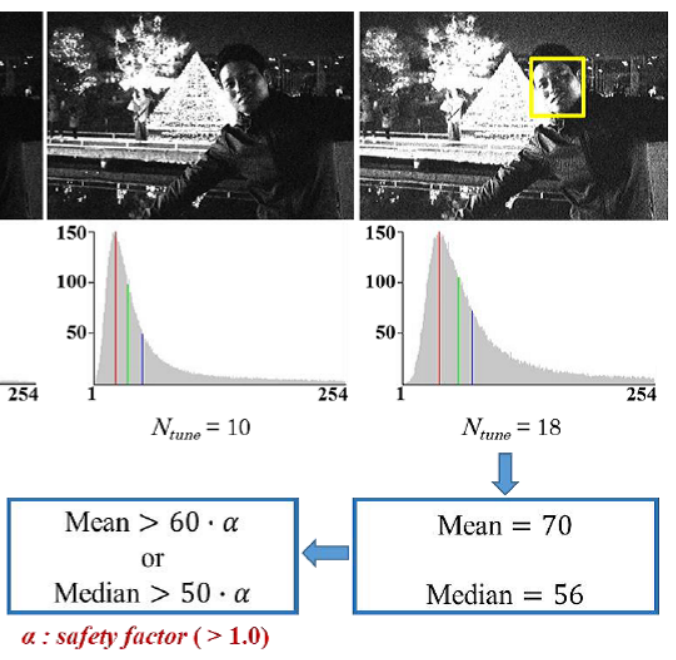

$N_{\text {tume }}=18$

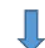

Mean $=70$

Median $=56$

Figure 3: Effect of the iteration number of Collins type SR; $N_{\text {tune }}$. 


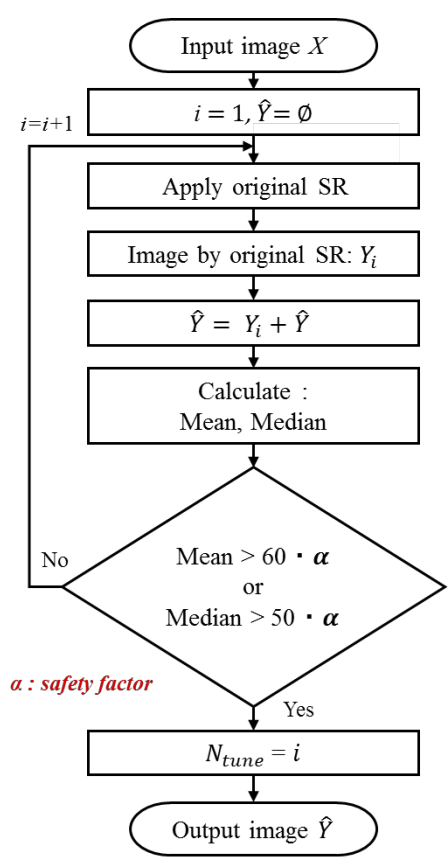

Figure 4: Proposed SR algorithm for image enhancement.

the value of $N_{\text {tune }}=18$. From experiments of Collins type $\mathrm{SR}$, face detection becomes possible when the condition of Mean $>60$ and Median $>50$ is satisfied. The factor $\alpha$ was introduced in order to allow the condition to have a margin and increase the detection rate. This factor is assumed to be 1 to 1.2 .

2.3 Proposed SR algorithm The proposed SR algorithm is the upgraded system of Collins type SR, where the SR parameters are automatically tuned instead of manual tuning. We have introduced the $N_{\text {tune }}$ auto-tuning mechanism in our proposed method. The flow of the proposed algorithm is shown in the Fig. 4 According to this flow diagram, $X$ is the original image, $Y$ is the image corrected or enhanced by using the SR technique, represents the $Y_{i}$ number of the SR process and $\alpha$ represents the safety factor for detection and set to 1.08. The same as in the previous section, the noise value is 3 , and the threshold value is 0 . By setting the threshold value to 0 , occurrence of a negative gradation value generated by the random noise can be excluded. This prevents from diminishing the important information in the dark images. Also, we have adopted Mean $>60 \cdot \alpha$ and Median $>50 \cdot \alpha$ described in Section 2.2. Fig. 5 shows the execution results of 12 cases applying the proposed SR algorithm.

\section{Experiment and evaluation of the proposed algo- rithm}

3.1 Preparation for experiments An auto-tuning system was developed based on the algorithm shown in the Fig. 4. In this system, Mean and Median are obtained after stochastic resonance processing. If Mean and Median satisfy the condition, the image enhancement process by using SR gives an output enhanced image. The safety factor $\alpha$ was introduced for the purpose of reducing the error of face detection for the case where the shooting condition is bad. Here, $\alpha$ for Mean and Median was set to 1.08 .

Proposed SR algorithm for image enhancement required output image due to the presence of many image pixels at 0 or 255 . We have developed a $\mathrm{C}$ programming code by using the Visual studio 2010 and the Open CV library on PC (i7-5820) using Windows 8.164 bit 0S. For face detection experiments, Lamsal's face detector [10] and Open CV face detection package [11] were used, and for detection of human body, upper body detection of Open CV human body detection package was used. The Open CV face detection package is based on V.J. face detector.

3.2 The results for Face detection We conducted an experiment to compare the proposed SR algorithm with the contrast adjustment method. Here we also compare the Lamsal's face detector with the Open CV face detection package. The results of the experiment are shown in Table 1.

When face detection was performed by the Lamsal's face

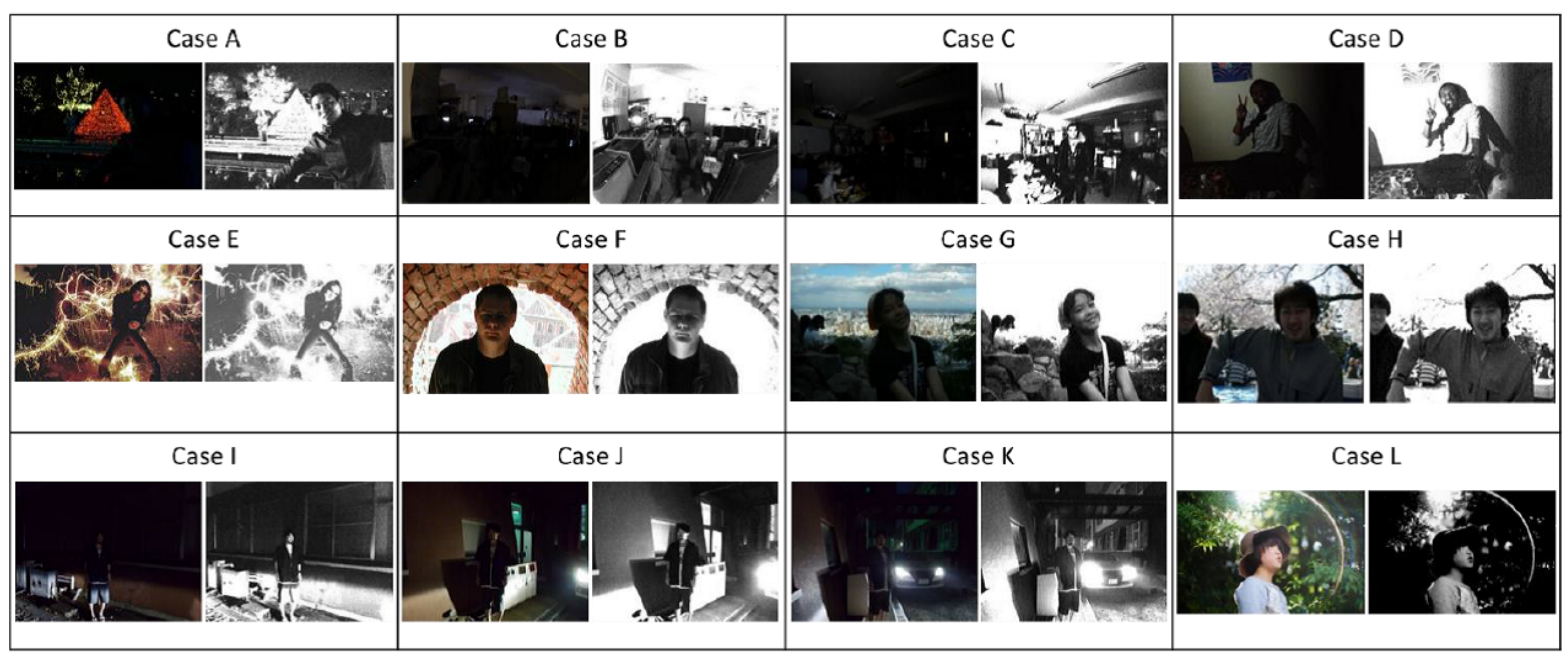

Figure 5: Execution of the proposed SR algorithm; Left is the input image, right is the output image for each case. 
Table 1: Comparison of face detection results.

\begin{tabular}{|c|c|c|c|c|c|}
\hline \multirow{2}{*}{ Photographing conditions } & \multirow{2}{*}{ Case \# } & \multicolumn{2}{|c|}{ Proposed algorithm using SR } & \multicolumn{2}{|c|}{ Contrast adjustment method } \\
\hline & & $\begin{array}{c}\text { Lamsal's face } \\
\text { detector }\end{array}$ & $\begin{array}{l}\text { V.J. Face detector } \\
(\text { OpenCV) }\end{array}$ & $\begin{array}{c}\text { Lamsal's face } \\
\text { detector }\end{array}$ & $\begin{array}{c}\text { V.J. Face detector } \\
(\text { OpenCV })\end{array}$ \\
\hline dark \& illmination variant & A & $\odot$ & $\Delta$ excess & $\times$ & $\times$ \\
\hline \multirow{3}{*}{ dark } & B & () & ○ & $\times$ & $\Delta$ excess \\
\hline & $\mathrm{C}$ & ๑ & $x$ & $\Delta$ excess & $\Delta$ excess \\
\hline & $\mathrm{D}$ & ๑ & $x$ & $\times$ & $\Delta$ excess \\
\hline illmination variant & $\mathrm{E}$ & () & $x$ & $x$ & $\times$ \\
\hline \multirow{3}{*}{ back light } & $\mathrm{F}$ & O & $x$ & $x$ & $x$ \\
\hline & G & ๑ & $x$ & $x$ & $x$ \\
\hline & $\mathrm{H}$ & ( ) & $\Delta$ shortage & $\Delta$ shortage & $\triangle$ shortage \\
\hline \multirow{3}{*}{ halation } & I & (๑) & $\times$ & $\times$ & $\times$ \\
\hline & $\mathrm{J}$ & (๑) & ๑) & $x$ & $x$ \\
\hline & $\mathrm{K}$ & ( ) & () & $x$ & $x$ \\
\hline flare or ghost & $\mathrm{L}$ & 0 & $\times$ & $x$ & $x$ \\
\hline \multicolumn{6}{|c|}{ ๑ : correct face detection and fulfilling exit condition of SR } \\
\hline \multicolumn{6}{|c|}{$\bigcirc:$ correct face detection but not fulfilling exit condition of SR } \\
\hline \multicolumn{6}{|c|}{$\Delta:$ excess or shortage of face detection } \\
\hline
\end{tabular}

Table 2: Comparison of human body detection results.

\begin{tabular}{|c|c|c|c|}
\hline \multirow{2}{*}{ Photographing conditions } & \multirow{2}{*}{ Case \# } & \multicolumn{2}{|c|}{ Human body detection(OpenCV) } \\
\hline & & Proposed algorithm using SR & Contrast adjustment method \\
\hline dark \& illumination variation & A & $\times$ & $\times$ \\
\hline \multirow{3}{*}{ dark } & B & () & $\triangle$ excess \\
\hline & $\mathrm{C}$ & ๑ & ๑ \\
\hline & $\mathrm{D}$ & ๑) & $\Delta$ excess \\
\hline illmination variant & $\mathrm{E}$ & $x$ & $\times$ \\
\hline \multirow{3}{*}{ back light } & $\mathrm{F}$ & () & (-) \\
\hline & G & ๑ & $\triangle$ excess \\
\hline & $\mathrm{H}$ & $\triangle$ shortage & $\triangle$ shortage \\
\hline \multirow{3}{*}{ halation } & I & $\odot$ & $\Delta$ excess \\
\hline & $\mathrm{J}$ & $\triangle$ excess & $\triangle$ excess \\
\hline & K & $\times$ & $\times$ \\
\hline flare or ghost & $\mathrm{L}$ & $\times$ & $\times$ \\
\hline \multicolumn{4}{|c|}{ ๑ : correct detection and fulfilling exit condition of SR } \\
\hline \multicolumn{4}{|c|}{$O$ : correct detection but not fulfilling exit condition of SR } \\
\hline \multicolumn{4}{|c|}{$\Delta:$ excess or shortage of detection } \\
\hline \multicolumn{4}{|c|}{$x:$ no detection } \\
\hline
\end{tabular}

detector, 10 out of 12 cases could be accurately detected. Impairment is two cases, and the detection rate is $80 \%$ or more. The proposed method is superior to the contrast adjustment method in all cases.

Also, using the proposed method, Lamsal's face detector is superior to the V.J. face detector.

3.3 The results for human body detection We carried out human body detection experiments using upper body detection package of Open $\mathrm{CV}$. The results are shown in Table 2.

From this table it can be seen that the proposed method is superior to the contrast adjustment method for human body detection. In the contrast adjustment method, excessive detection of human bodies occur frequently.

3.4 Comparison with contrast adjustment process In order to validity the effectiveness of our proposed system, we compared our proposed system with the contrast adjustment process under various difficult illumination conditions.

As shown in the Fig. 6, case $\mathrm{C}$ to be shown later, the human in the image along with the face is detected by using our proposed algorithm as well as by the contrast adjustment process. If we look clearly at the contrasted adjusted images after applying the detector, we can see the detected objects rather than the face and the human, which can be regarded as error. It is because, the contrast adjustment method is a process of expanding the width of the histogram and converting the density. As we can see the histograms shown in the Fig. 6 is the result of non-continuous performance of the interpolation between the gradations values are not performed continuously.

But our proposed method the interpolation between the gradations values are performed continuously, which results in the proper histogram and clearly enhanced images. Since face detector and human detector are a method of detecting 


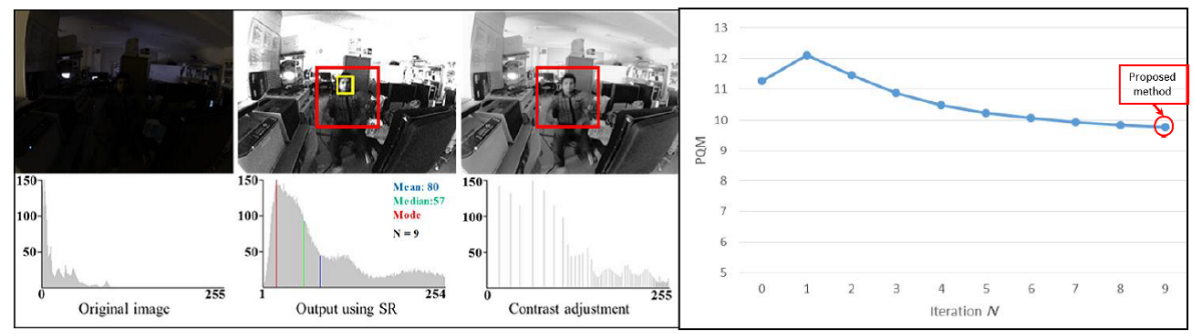

Case B

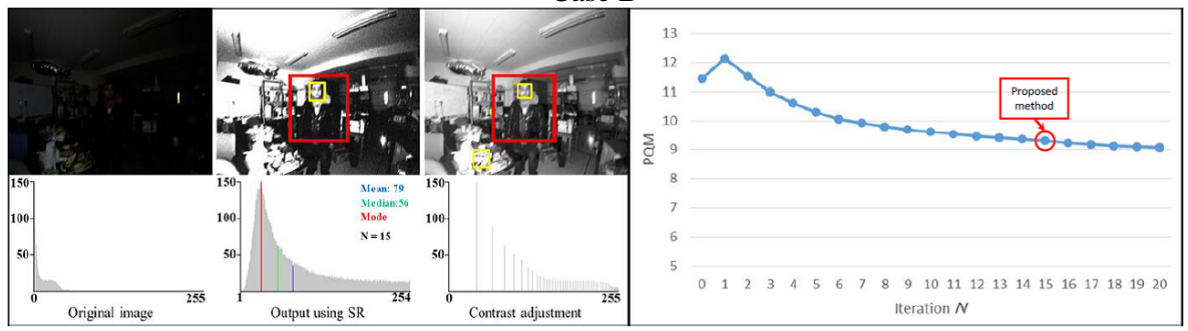

Case C
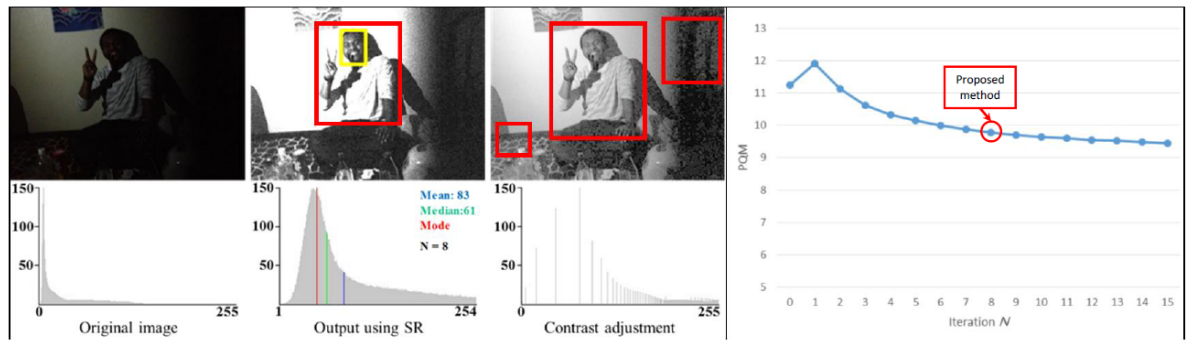

Case D
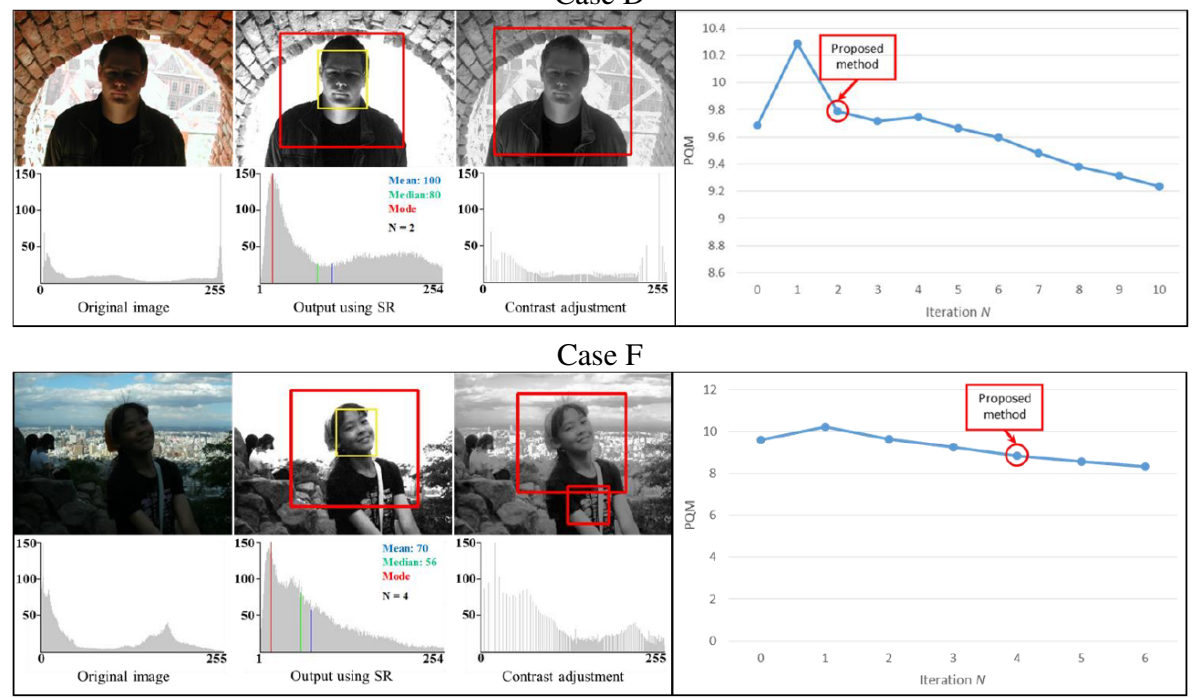

Case G

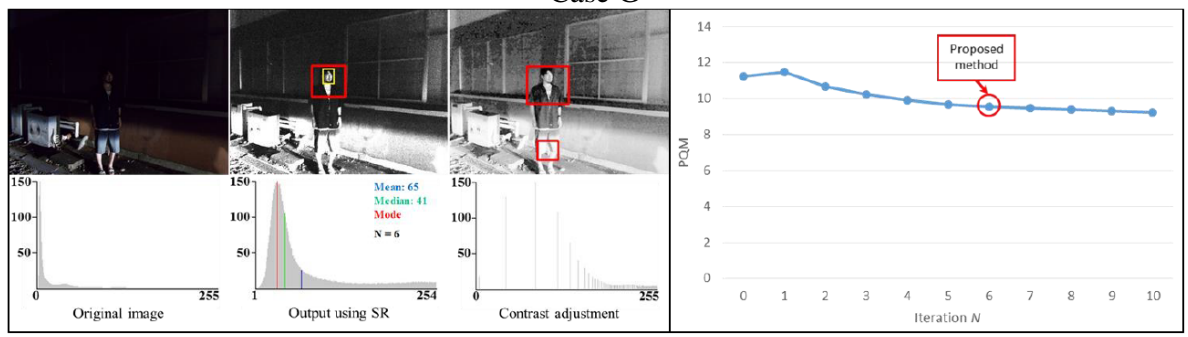

Case I

Figure 6: Comparison of the detection result of our Proposed SR algorithm and PQM. Face: yellow box, Upper body: red box 


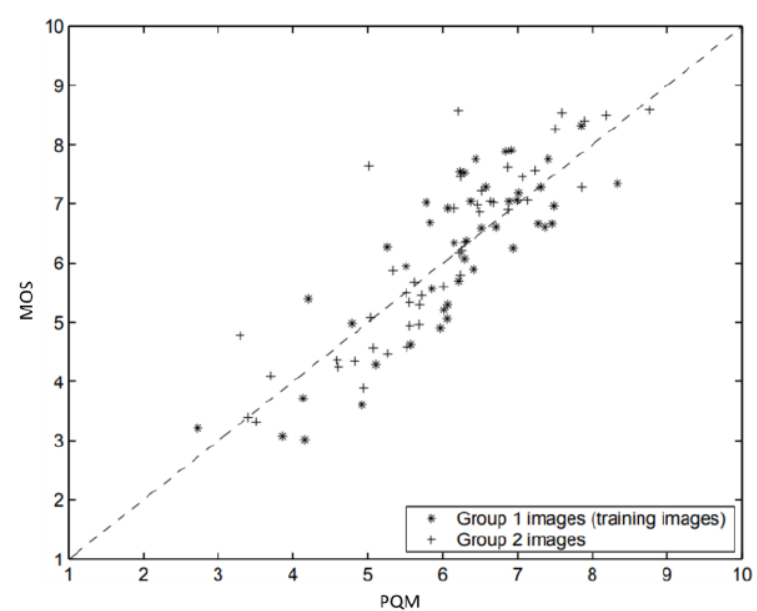

Figure 7: An example of relation between PQM and MOS (Ref.[12])

a feature pattern of the face and the human body from the contrast difference, we think that our proposed method is effective.

The proposed method improves excessive detection and insufficient detection compared with the contrast adjustment method.

\section{Evaluation of the proposed method based on com- parison with PQM of images}

A method of evaluating images without using face and human detectors is also necessary. Experiments were conducted using Perceptual Quality Metric (PQM) (12) as an evaluation method to apply the proposed method to various fields. A MATLAB function was used to calculate PQM for an image.

4.1 About PQM In addition to face and human body detection, a common evaluation criterion is required so that it can be applied to various detection. PQM is a metric that quantifies image quality. One to ten grades are evaluated, ten is high quality. This enables better quality measurement compared to subjective evaluation value, i.e., MOS (Mean Opinion Score). Fig. 7 shows that there is a high correlation between PQM and MOS.

4.2 Results of the face detection adding the body detection; comparison with PQM For the six cases in Fig. 5, experiments were conducted to compare the results of the proposed method with PQM. Results of detection of faces are and upper body yellow boxes. Results of detection of faces and upper bodies are indicated by yellow and red boxes respectively.

- Result of the case B:

The value of PQM was closest to 10 when $N=3$. Face was detected only by the proposed method at $N=9$. Body was detected by both methods, i.e., the proposed and contrast adjustment.

- Result of the case C:

The value of PQM was closest to 10 when $N=6$. Face and body were detected by the proposed method at $N=15$. In contrast adjustment method, there was erroneous detection.

- Result of the case D:

The value of PQM was closest to 10 when $N=6$. Face and body were detected by the proposed method at $N=8$. Using contrast adjustment, face detection was not possible, and body detection was erroneously detected.

- Result of the case F:

PQM was closest to 10 when $N=2$. In this case, the proposed method and PQM agreed. Face and body were detected by the proposed method at $N=2$.

- Result of the case G:

The value of PQM was closest to 10 when $N=1$. Face and body were detected by the proposed method at $N=4$.

- Result of the case I:

The value of PQM was closest to 10 when $N=4$. A face and a body were detected by the proposed method at $N=6$.

4.3 Summary of experiment results based on comparison with PQM In Five cases out of 12 cases of face detection, values of $N$ of the proposed method and PQM = 10 coincided.

Even in cases where they do not match, the difference between them is considered to be within an acceptable range.

PQM evaluates images globally, while face or human body detection evaluates faces of the images locally. This is the reason for not coinciding on the above several cases.

\section{Discussion and Conclusion}

We proposed an image enhancement method using stochastic resonance (SR), which auto tunes the parameters related to the SR and clarified the effectiveness of the proposed method by the experiments. The experiments were conducted using various images with severe shooting conditions, such as dark, illumination variant, back lighting, halation, flare and ghost.

Unlike from the contrast adjustment process, the histogram is not only extended but are interpolated as well. Since the histogram is interpolated, the contrast difference was enhanced. The proposed method improves excessive detection and insufficient detection compared with the contrast adjustment method.

Based on the face detection and human detection experimentation, the proposed method showed a possibility of reducing the detection error in compared to the contrast adjustment process.

The auto-tuning SR based image enhancement was developed to work on images with severe shooting conditions. Our proposed algorithm does not require manual tuning of SR parameters for images of various shooting conditions.

In case of the human detection, it was necessary to measure the degree of image enhancement by using the PQM. To detect objects other than the face and the human body, 
quality evaluation metrics of the entire image such as PQM will be required.

The system can be applicable in the various fields that uses images. Also, it will help to increase the effectivity of the image processing system that depends on the image enhancement.

\section{References}

[1] B. Lamsal, N. Kojima and N. Matsumoto, "Impact of the stochastic resonance on dark and illumination variant images for face detection", Journal of the Institute of Industrial Applications Engineers, vol.3, no.4, pp.167-173, 2015.

[2] N. Kojima, B. Lamsal and N. Matsumoto, "An adaptive tuning stochastic resonance approach for image enhancement on illumination variant images", Journal of the Institute of Industrial Applications Engineers, vol.5, no.1, pp.1-8, 2017.

[3] N. Kojima, N. Matsumoto and M. Yamashiro, "Image enhancement under severe lighting conditions using stochastic resonance", LIFE2017, 2017. (in Japanese)

[4] E. Simonotto, M. Riani, S. Charles, M. Roberts, J. Twitty and F. Moss, "Visual perception of stochastic resonance", Phys. Rev. Lett., 78(6), pp.1186-1189, 1997.

[5] R. Chouhan, R. K. Iha and P. K. Biswas, "Wavelet-based contrast enhancement of dark images using dynamic stochastic resonance", ICVGIP' 12, Mumbai, India, 2012.

[6] R. K. Jha and R. Chouhan, "Noise-induced contrast enhancement using stochastic resonance on singular values", SIViP 8 , pp.339-347, 2014.

[7] R. Benzi, A. Sutera and A. Vulpiani, "The mechanism of Stochastic Resonance", J. Physics. A: Math and General, vol.14, pp. L453 -L457,

[8] J. J. Collins, C. C. Chow and T. T. Imhoff, "Stochastic resonance without tuning", Letters to NATURE, vol.376, pp.236238, 1995.

[9] S. Kasai, K. Miura and Y. Shiratori, "Threshold-variation enhanced adaptability of response in a nanowire field-effect transistor network", Applied Physics Letters, Vol.96, No.19, pp.194102, 2010.

[10] B. Lamsal and N. Matsumoto, "Effects of the Unscented Kalman filter process for high performance face detector", Intl. J. of Information and Electronics Engineering, Vol.5, No.6, pp.454-459, 2015.

[11] http://opencv.jp/

[12] Z. Wang, H. R. Sheikh and A. C. Bovik, "No-reference perceptual quality assessment of JPEG compressed images", Proc. IEEE Int. Conf. Image Processing, vol.1, pp.477-480, 2002.

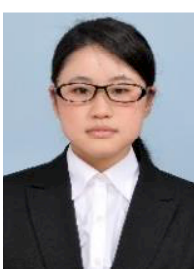

Noriko Kojima (Member) was born in Gunma, Japan. She received her bachelor degree in System and Information engineering and master degree in Information science and Manufacturing engineering from the Ashikaga Institute of Technology, Tochigi, Japan in March 2014 and March 2016 respectively. Currently she is a Ph.D. student in Ashikaga Institute of Technology since April 2016. Her research interests includes Image processing, Computer vision and Image recognition.

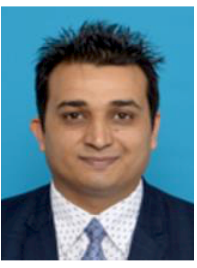

Bikash Lamsal (Member) received his Doctoral degree of Engineering from Ashikaga Institute of Technology, Tochigi, Japan in March 2017. Currently he is working as a researcher in Kajima Technical Research Institute, Tokyo, Japan. He is the member of the ACM and IIAE. His research interest includes image processing, computer vision and VR/AR applications.

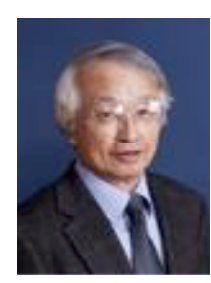

Naofumi Matsumoto (Member) received his doctoral degree of engineering from Sophia University, Tokyo, Japan in 1978. Currently, he is a professor emeritus at Ashikaga University (changed from AIT). He engaged in education and research for 42 years at the Faculty of Engineering of Sophia University and AIT. He is the member of JSME, IIAE, JIMA, ORSJ and Information processing society of Japan. His research interest includes system optimization, soft-computing and image processing.

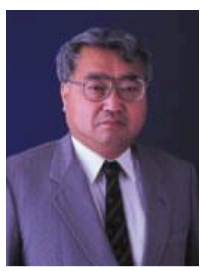

Mitsuo Yamashiro (Non-member) received the doctoral degree of engineering from Keio University, Japan, in 1990. Currently, he is a Professor in the division of systems and information engineering at Ashikaga University. $\mathrm{He}$ is the member of JSME, IEEJ and JIMA. His research interests are in the theory and applications of manufacturing systems engineering and powder technology. 